\title{
MD simulation of the effect of contact area and tip radius on nanoindentation
}

\author{
CHEN Shangda ${ }^{1,2}$ \& KE Fujiu ${ }^{1,2}$
}

1. Department of Applied Physics, Beijing University of Aeronautics and Astronautics, Beijing 100083, China;

2. State Key Laboratory of Non-linear Mechanics (LNM), Institute of Mechanics, Chinese Academy of Sciences, Beijing 100080, China

Correspondence should be addressed to Chen Shangda (email: chensd@Inm.imech.ac.cn)

Received August 27, 2003

\begin{abstract}
Molecular dynamics simulations of nanoindentation are performed on monocrystal copper. A new "contact atoms" method is presented for calculating the contact area. Compared with conventional methods, this method can provide the contact area more accurately not only for sink-in but also for pile-up situation. The effect of tip radius on indentation is investigated too. The results indicate that the measured hardness of the material will become higher as the tip radius increases.
\end{abstract}

Keywords: nanoindentation, molecular dynamics, contact area, zero point of penetration depth, nanohardness.

DOI: $10.1360 / 03 y w 0163$

Nanoindentation, namely depth-sensing indentation (DSI), involves forcing a rigid indenter with known geometry into the surface of a material while continuously monitoring the load on the indenter, the displacement of the indenter into the surface, and the time of the experiment. The depth is then used to calculate the projected area of contact for the purpose of calculating the hardness and elastic modulus. In fact, various errors are associated with this procedure. One of them comes from the measurement of the penetration depth. Ideally, the penetration depth should be calculated from the free surface of a specimen as zero point. However, how to correctly identify the free surface from the measurement of load-displacement is a real problem. The effect of incorrect zero point of penetration depth on the hardness will become even severe in shallow nanoindentation $^{[1,2]}$. It is well known that the Oliver and Pharr formula ${ }^{[3]}$ is almost always used to calculate hardness in nanoindentation. However this will lead to some confusion in the data of nano-hardness. In fact their model is based on the elastic assumption and suitable for sink-in materials only. As for pile-up materials, Oliver's method may bring about great errors ${ }^{[4]}$. Since shallow nanoindentation involves only a few atomic layers near the surface, continuum approach to the problem becomes questionable. Accordingly, molecular dynamics (MD) simulation is adopted in the 
present study.

A lot of atomistic simulations have been carried out in the investigation of nanoindentation ${ }^{[5-9]}$. Landman et al. ${ }^{[5]}$ found the "jump-to-contact" phenomenon. Leng et al. investigated very shallow nanoindentation $(1-2 \text { atomic layers })^{[6]}$. Their simulations show that the material's response was linear elastic during the loading-unloading cycle and the contact stresses were comparable with those calculated with macroscopic Hertzian theory. Gannepalli et al. simulated the plastic deformation during silicon nanoindentation $^{[7]}$. Cheong et al. investigated the phase transformations in silicon monocrystals due to nanoindentation ${ }^{[8]}$. Christopher et al. simulated the pile-up of iron and silver during nanoindentation ${ }^{[9]}$. Unfortunately, the contact area and the tip radius that affect shallow indentation very severely have not been closely examined. Although there are some reports ${ }^{[10,11]}$ on the effect of pile-up and tip radius by finite element method (FEM), the results may be questionable for shallow indentation because of the limitation of continuum. So it is significant to investigate the effect of contact area and tip radius on nanoindentation by means of MD simulation. We focus on MD simulations on the contact area on shallow indentation. In order to calculate contact area correctly, we propose "contact atoms" method. Then the effect of tip radius is investigated.

\section{1 “Contact atoms" method in MD simulation}

In our MD simulations, the sample and indenter are monocrystal copper and rigid diamond respectively, shown in fig. 1 . The sample is modeled as a finite slab $\left(32 a_{0} \times\right.$ $32 a_{0} \times 16 a_{0}$ ) with 65536 atoms in a face-centered cubic (fcc) lattice, where $a_{0}$ is the lattice constant of copper $(0.3615 \mathrm{~nm})$. The top (001) surface of the sample is assumed to be stress free. Periodic boundary conditions are used in two transverse ( $x$ and $y$ ) directions. Two layers of atoms at the bottom of the sample are fixed to prevent the sample from moving vertically in the indentation process. A hemispherical diamond tip with radius $6 a_{0}$ is adopted to indent into the copper monocrystal sample.

First, we briefly introduce the potentials used in our simulation. The wellestablished embedded atomic method (EAM), which has been very successful in modeling elastic properties, defect formation energy and fracture mechanisms of various close-packed bulk metals ${ }^{[12,13]}$ is adopted for the copper sample. The relevant parameters of copper provided in ref. [14] are used. The interaction between the copper atoms and the diamond indenter atoms is modeled by the Morse potential ${ }^{[15]}$.

Initial velocities of copper atoms are assigned from the Maxwell distribution. In order to suppress the effect of the thermal fluctuation, the temperature in the simulations is kept very low, namely $1 \mathrm{~K}$. The Newton's equations of motion for the sample atoms are numerically integrated using the Leap-Frog algorithm ${ }^{[16]}$ with a fixed time step of 10 fs. At the beginning of each simulation, the tip of the indenter is sited $1 a_{0}$ above the middle of the sample surface and the maximal penetration depth is set at $5 a_{0}$ (10 atom layers). The average indentation speed is about $30 \mathrm{~ms}^{-1}$, several orders of magnitude 


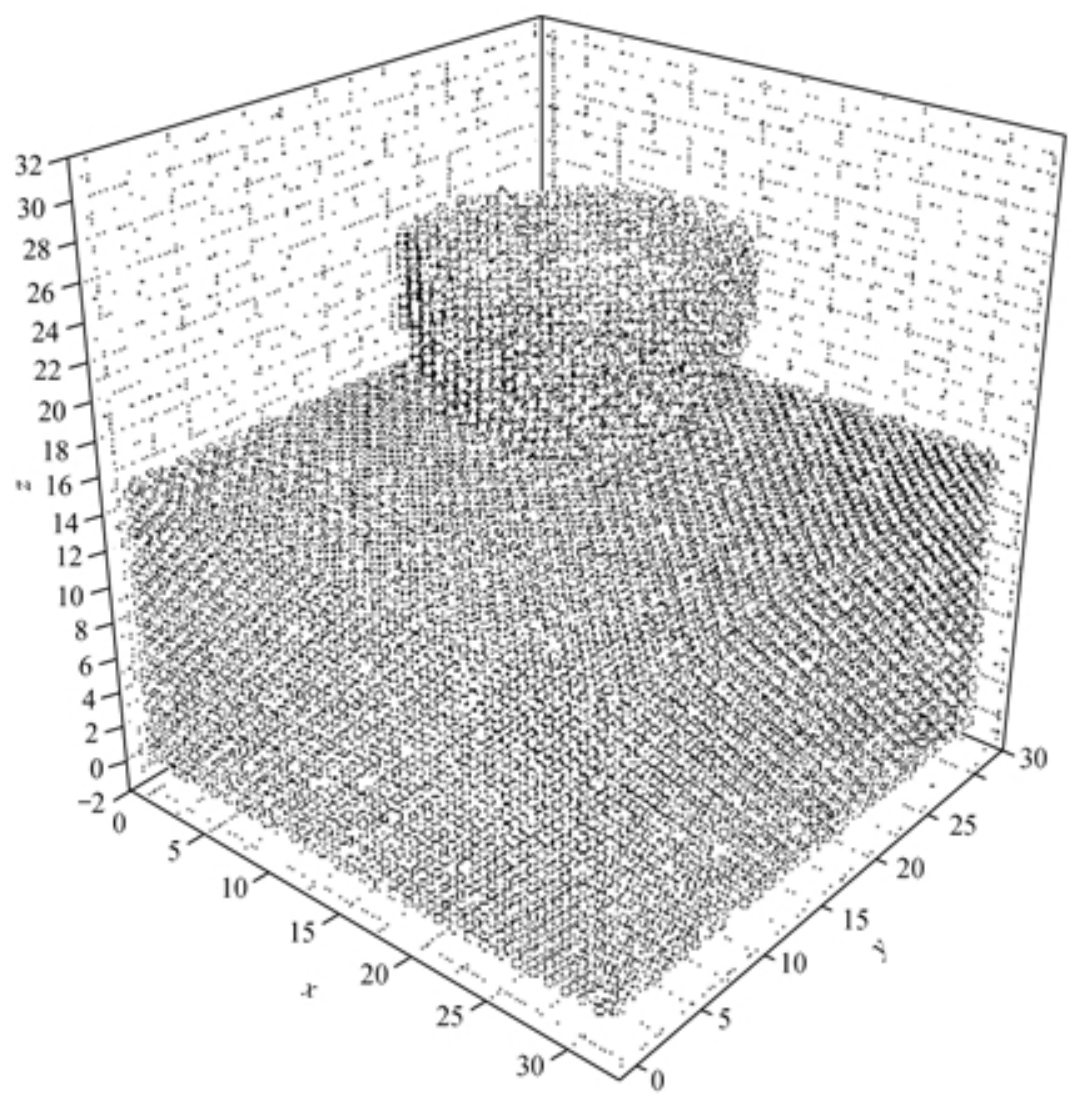

Fig. 1. Initial configuration of the tip and the sample.

faster than the experimental one, the typically $10^{-9}-10^{-8} \mathrm{~ms}^{-1}$.

Now, we propose a new "contact atoms" method to calculate the projected contact area of nanoindentation. When the tip enters the sample, an interface between copper and carbon atoms will form. From the force versus distance curve of the Morse potential of copper and carbon atoms (fig. 2(a)), we can see that only when the distance between two atoms becomes less than $r_{0}$, does the force become repulsive. So, if the distance between a carbon atom and any copper sample atoms is less than $r_{0}$, we define that carbon atom as a "contact atom". Fig. 2(b) shows all contact atoms and their projection on $x-y$ plane when the penetration depth is about $5 a_{0}$. Then we can calculate the projected area of the "contact atoms" at every time step when simulations proceed.

In our algorithm, two approaches were applied to the calculation of contact area. First, since the diamond tip is rigid hemisphere, the projection of all contact atoms would approximately be a circle (fig. 2(b)). Then we work out the radius $(R)$ of the projection easily. Since every tip atom has an action range, which is about $r_{0}$, we will add $r_{0}$ to $R$ as the radius to calculate the projected contact area by the formula: Area $=\pi \times$ $\left(R+r_{0}\right)^{2}$. In order to check the validity of the approximation, another method is used. 


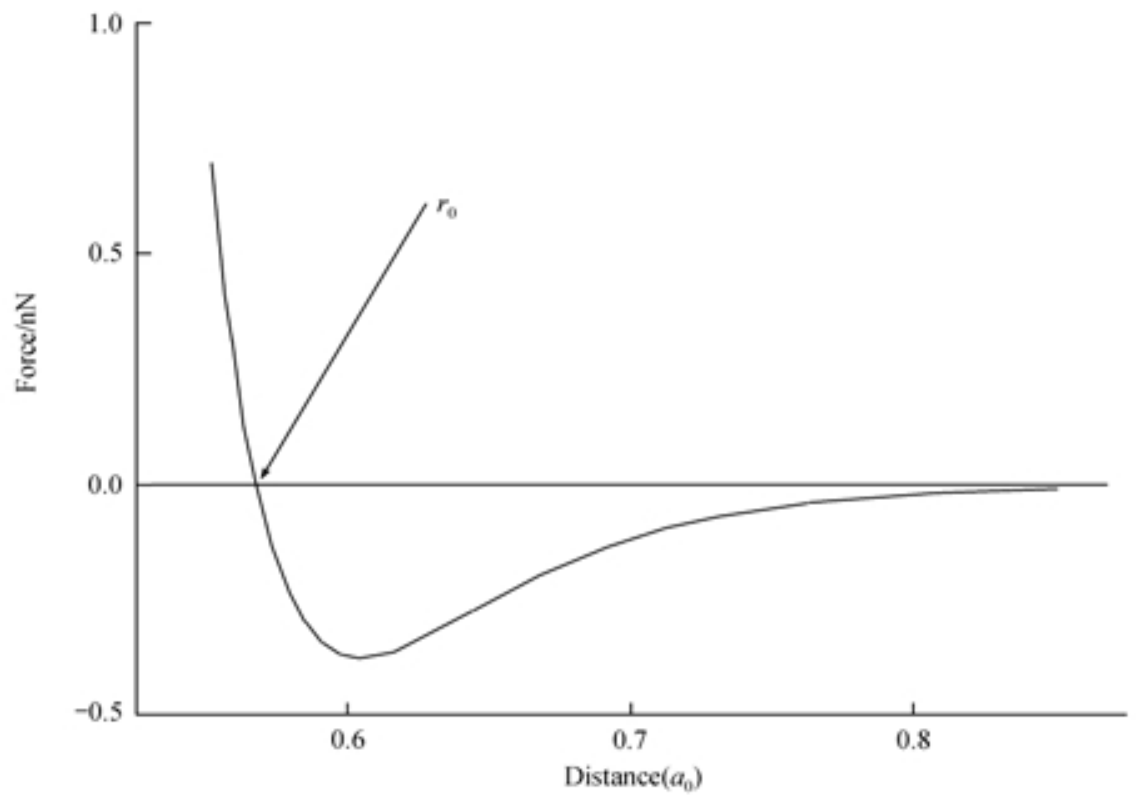

(a)

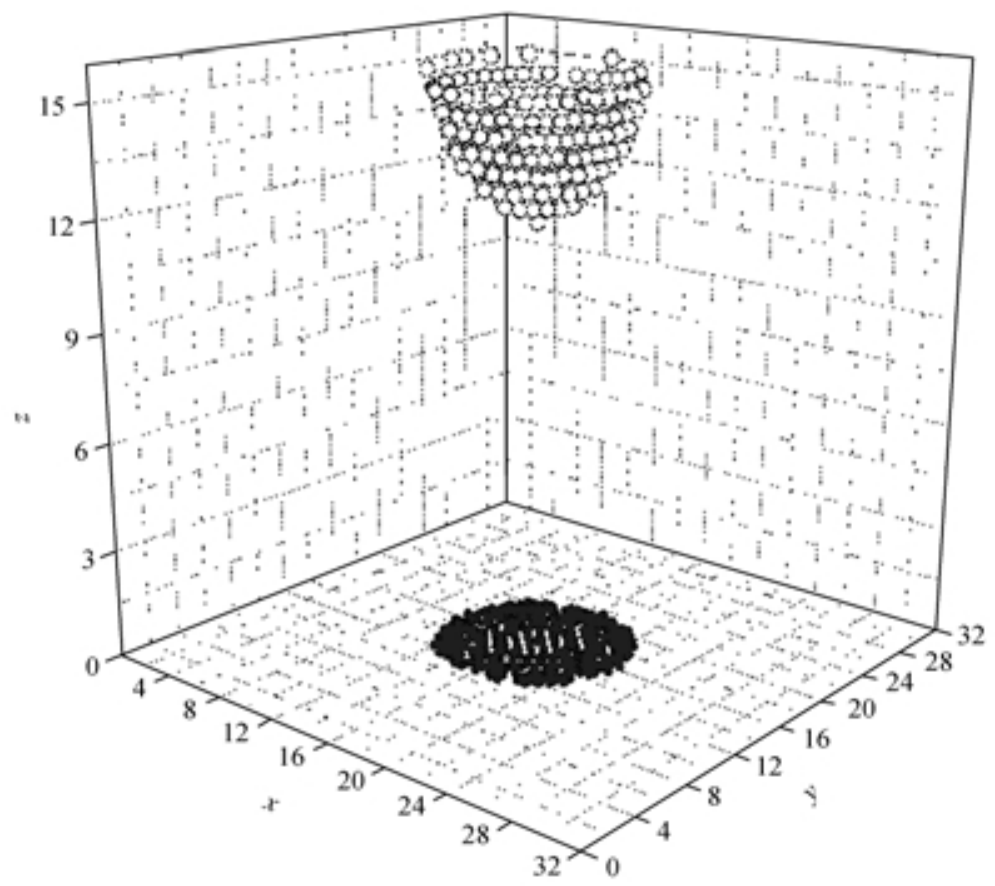

(b)

Fig. 2. (a) Force versus distance curve between a copper atom and a carbon atom; (b) snapshot of all contact atoms and the projection.

Using the modified version of triangle ${ }^{[17]}$ to connect all atoms of the projection to form a 
polygon, we can calculate the area of the polygon. Comparing these two methods, we found that the areas are very close to each other at the same depth. For instance, when the depth is about $3 a_{0}$, the difference is less than $3 \%$. So, the assumption of a circular projection is a reasonable approximation. It should be emphasized that the area calculated by the "contact atoms" method is a physical contact area. Unlike Oliver-Pharr formulas, the method is independent of the calculation of depth and the assumption of deformation modes, such as sink-in and pile-up. Hence, the hardness obtained using this method is a truly material response.

\section{Results and analysis}

\subsection{The effect of contact area}

The force versus displacement curve for the whole cycle of indentation is shown in fig. 3(a). The force, $F_{z}$ is calculated as the sum of the normal component of the forces exerted on the tip atoms by the sample. In order to calculate the penetration depth and contact area which are used to calculate the hardness, one needs to determine the zero point of penetration depth beforehand. There are three methods to calculate the zero point of penetration depth and contact area:

(1) Pure MD representation: Once a tip atom is first repulsed by the sample, it is defined to be the zero point of penetration depth. Then use "contact atoms" method to calculate the projected contact area.

(2) Hybrid representation: Once the force on the tip first turns to become repulsive from attractive, it is defined to be the zero point of penetration depth. Then use "contact atoms" method to calculate the projected area.

(3) Macroscopic representation: Once the force on the tip first turns to become repulsive from attractive, it is defined to be the zero point. Then use Oliver-Pharr method $^{[3]}$ to calculate the project contact area.

For the sake of comparison with experiments, later, we adopt the hybrid representation and the macroscopic representation only.

The initial stage of the force-depth curve is shown in fig. 3(a). From this figure one can see that there are two points where the force turns from attractive to repulsive: point $A$ and point $B$. Why? It is because the tip is hemispherical. When the atoms of the tip get close to the sample, the tip is attracted by the sample and the total force on the tip is attractive. As the tip gets even closer to the sample, the top atoms are repulsed by the sample, but all other atoms are still attracted by the sample. At point $A$ the total force starts to turn repulsive from attractive. As the depth increases, more and more tip atoms are attracted by the sample, so the total force turns attractive again. When the depth reaches point $B$, repulsive force becomes dominant and can never reverse.

Fig. 4 shows three area-depth curves by selecting $A$ as zero point of penetration depth. Obviously, the areas calculated by pure MD representation and hybrid 
representation respectively have a small shift in depth. The stepwise area-depth curves in these two representations are due to the nature of contact atoms method. That is to say, when the tip penetrates one atom layer the contact area changes. One important point in fig. 4 is that the two representations calculated with "contact atoms" method generally show larger contact area than that obtained with Oliver-Pharr method. When the depth is $1.5 \mathrm{~nm}$, the area calculated by hybrid representation is $20 \%$ more than that calculated by macroscopic representation. We will explain this later.

Fig. 5 shows two hardness curves. The negative hardness comes from the attractive force between points $A$ and $B$ (fig. 3(b)). The depth is only several angstroms and the
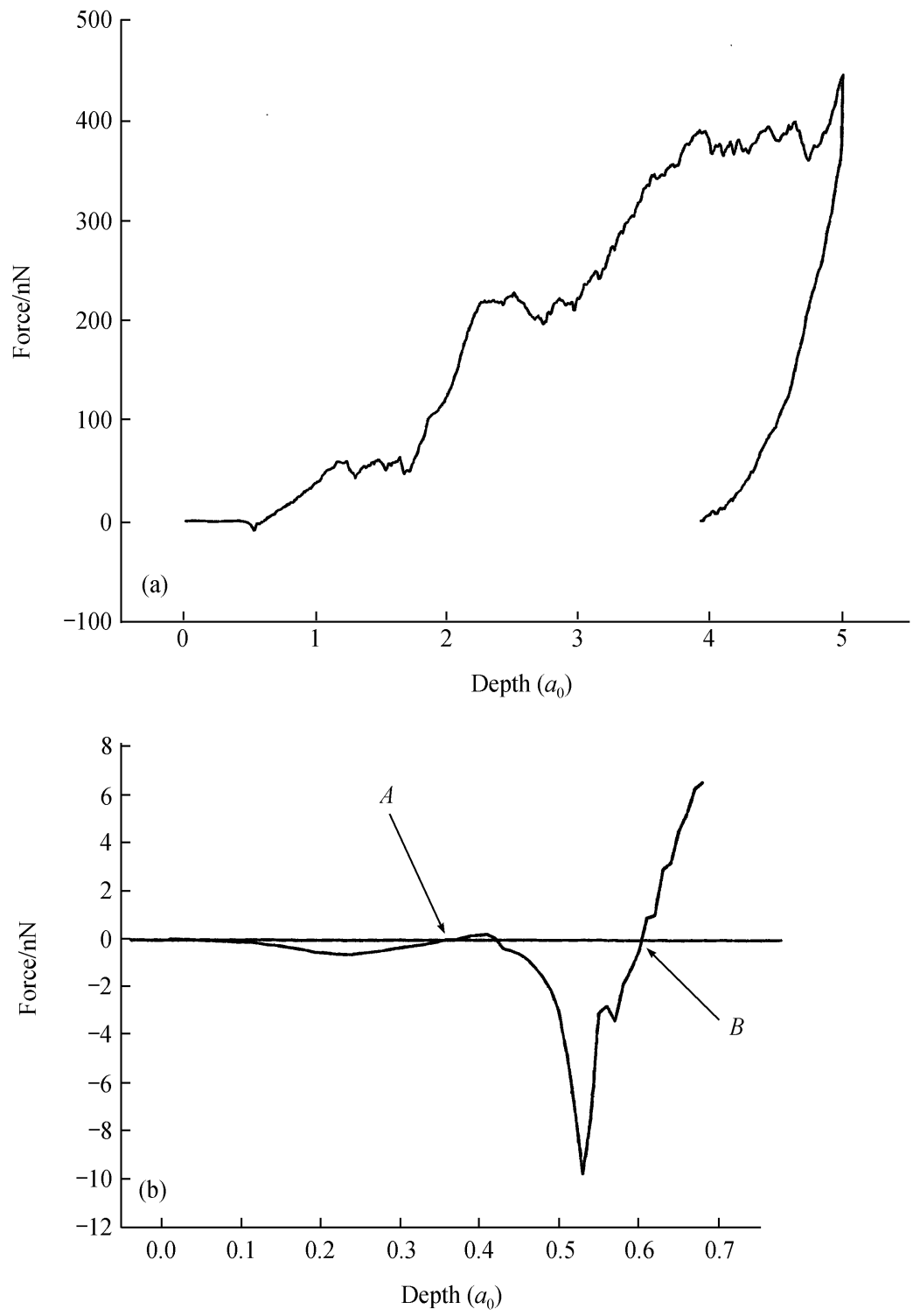

Fig. 3. (a) Force versus depth curve; (b) initial stage of force versus depth curve. 


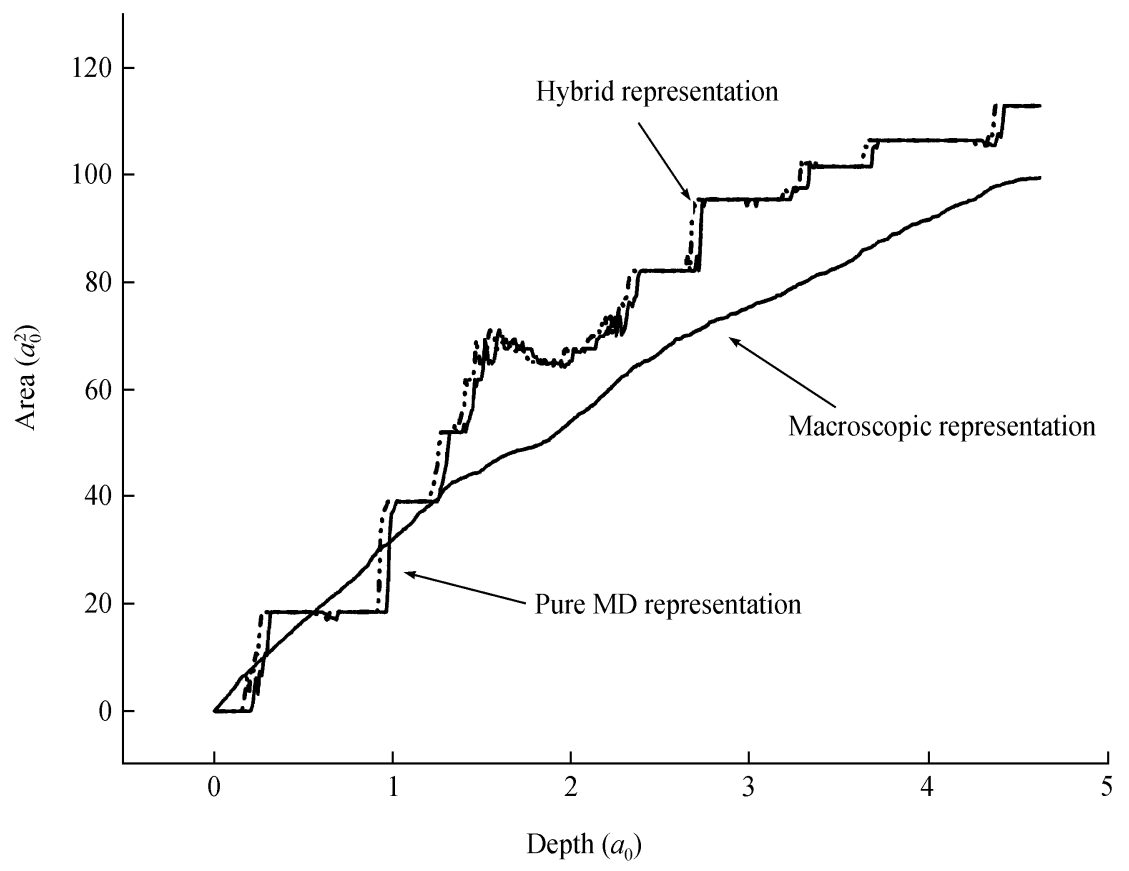

Fig. 4. Area versus depth curves.

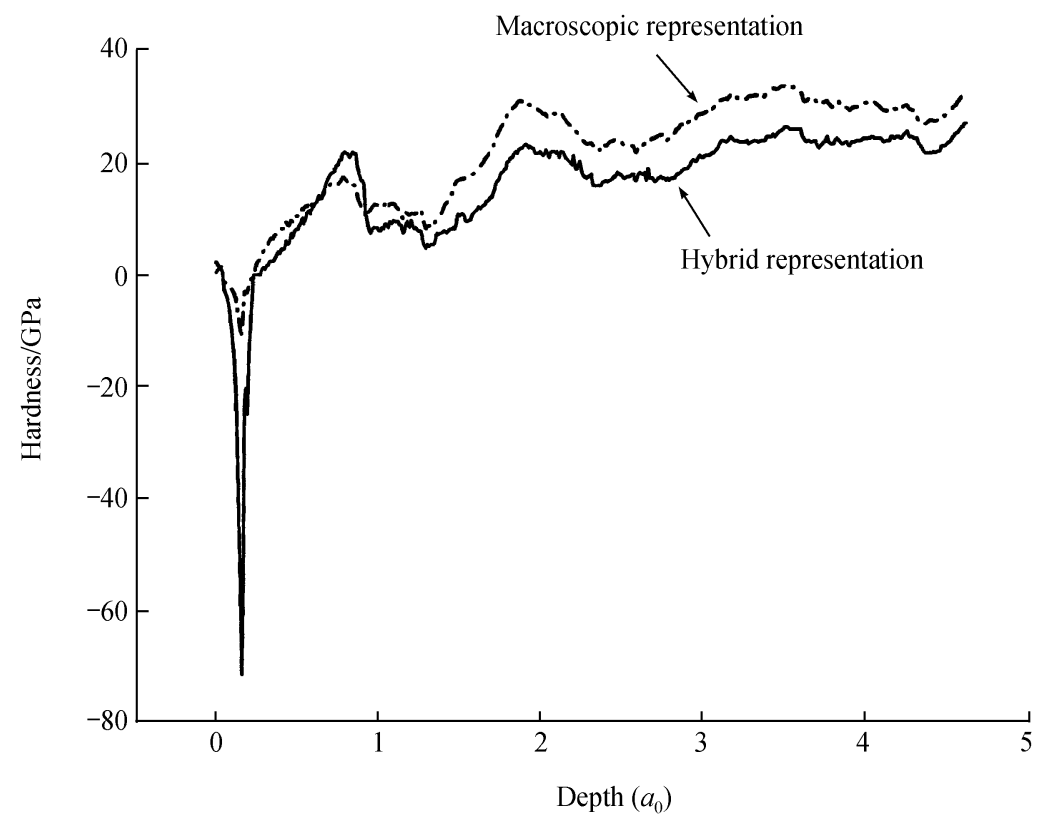

Fig. 5. Hardness versus depth curves.

hardness is meaningless. In fig. 5 the hardness calculated by the macroscopic representation is larger than that calculated by the hybrid representation with the increasing depth. When the depth is $1.5 \mathrm{~nm}$, the hardness calculated by the macroscopic representation is about $20 \%$ larger than that calculated by the hybrid representation. 
The physical reason for larger contact area and lower hardness obtained by the hybrid representation is pile-up of materials (see fig. 6). It is well known that Oliver and Pharr formula is based on elastic assumption and suitable for sink-in materials only. If the nanoindentation demonstrates pile-up situation, Oliver's method will underestimate the contact area and result in the overestimation of hardness. Bolshakov et al. ${ }^{[10]}$ investigated the influence of pile up by FEM. They found that the materials piled up when $h_{\mathrm{f}} / h_{\max }>0.7$, where $h_{\mathrm{f}}$ is the residual depth and $h_{\max }$ is the maximum depth. According to Bolshakov's result, when $h_{\mathrm{f}} / h_{\max }$ is close to 1 , the Oliver-Pharr method overestimates the hardness by as great as $60 \%$. In our simulation, the value of $h_{\mathrm{f}} / h_{\max }$ is about 0.8 (see fig. 3(a)). The monocrystal copper is a pile-up material. By recording the coordinate of atoms, we can clearly see that some atoms are piled up along the periphery of the tip. Fig. 6 shows the piled up atoms when the depth is about $1.5 \mathrm{~nm}$.

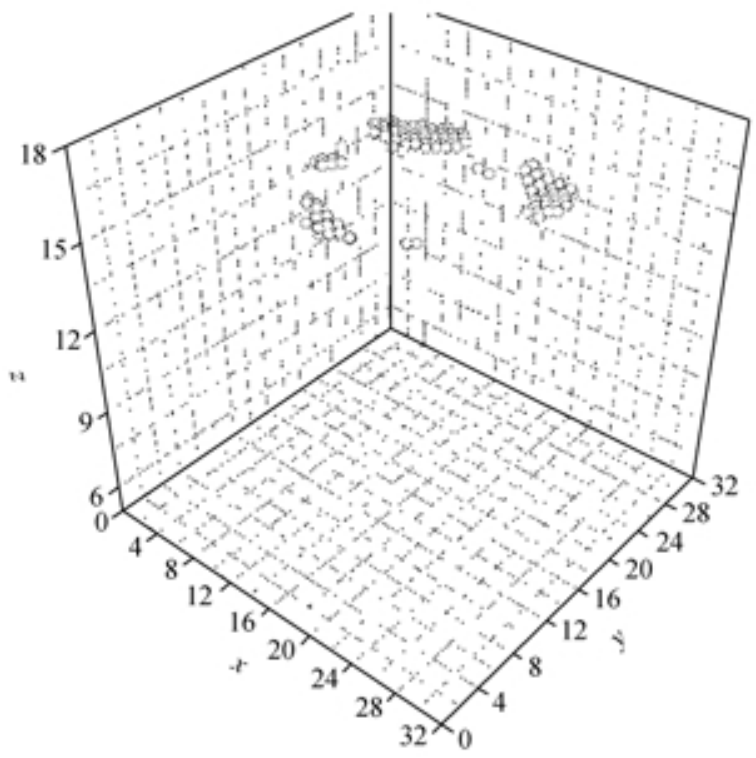

Fig. 6. Snapshot of pile-up atoms.

Then we analyze the selection of zero point of penetration depth. According to the final draft international standard of ISO/FDIS 14577-1, the following method is recommended: The zero-point is the touch point determined during the first increase in either the test force or the contact stiffness ${ }^{[18]}$. Looking back at the adopted zero point $A$, we found the repulsive force just after $A$ may be too small $(<1 \mathrm{nN})$ to monitor in experiment. In fact, we found only one transit point in our experiment. This is most probably the point $B$. Fig. 7(a) shows the hardness calculated by the hybrid representation when selecting different points as the zero point. The two curves are nearly the same except for a small shift. It is because the "contact atoms" method does not depend on the depth. Fig. 7(b) shows the hardness calculated by the macroscopic representation. From the figure one can see that the hardness calculated by selecting point $B$ as zero point is higher than that calculated by selecting point $A$ at the initial stage 

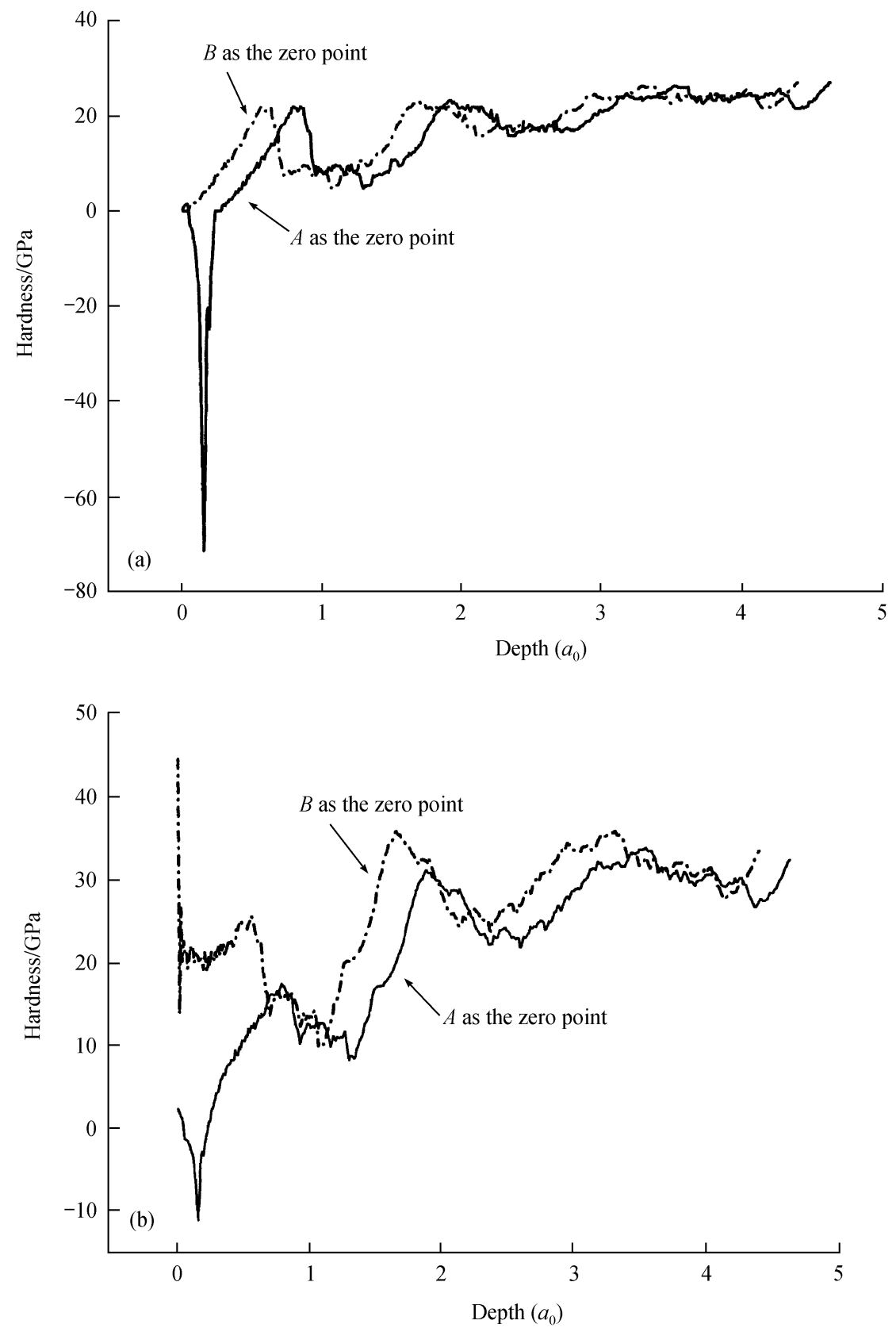

Fig. 7. (a) Hardness versus depth curve calculated by the hybrid representation; (b) hardness versus depth curve calculated by the macroscopic representation.

of nanoindentation. As the depth increases, the difference will become small, because Oliver's method depends on the penetration depth, namely the selection of zero point.

In summary, the proposed "contact atoms" method can give the contact area accurately and unlike Oliver-Pharr formulas, is independent of the measurement of 

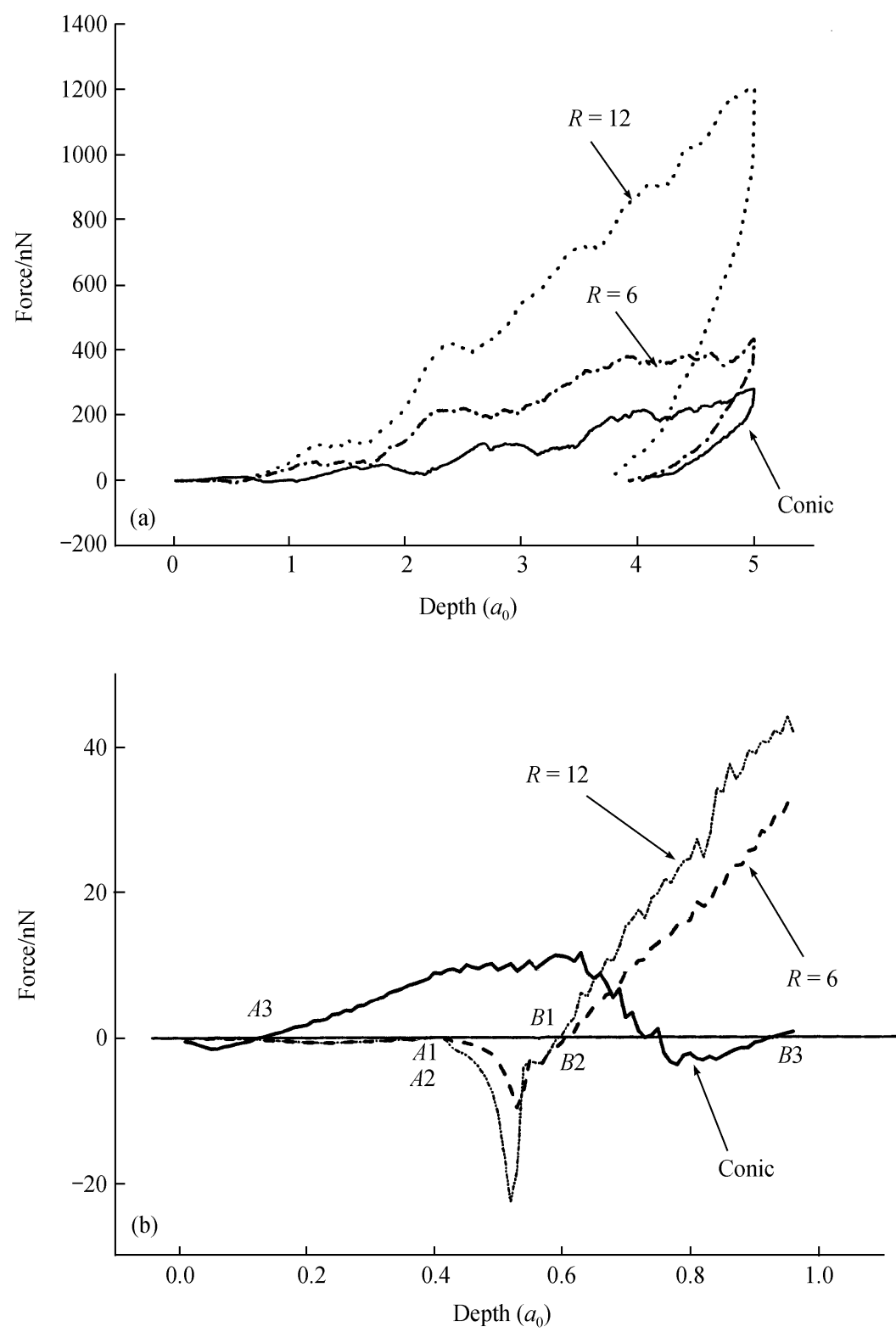

Fig. 8. (a) Force versus depth curves; (b) initial stage of force versus depth curves.

depth. Moreover, the contact atoms method may be suitable not only for sink-in but also for pile-up cases. Also, it is very easy to realize in MD simulation. However, how to realize the method in practical experiment still remains open.

2.2 The effect of the tip radius

In experiment the radius of the tip can be less than $40 \mathrm{~nm}$ originally. However the tip will become more and more blunt because of wear. In order to decrease the errors brought by the change in the tip radius, we usually have to correct the area function. But 
what actually happened in physics is still unknown. We investigated the effect of different radii of the tip by means of MD simulation. The tip radii are $6 a_{0}$ and $12 a_{0}$, respectively. In addition, a conic tip is also simulated.

Fig. 8(a) gives the force-depth curves for the three tips. At the same depth, the forces increase obviously with the increase in tip radius. In all of these curves, there are also two zero points: point $A$ and point $B$ (fig. 8(b)).

The hardness-depth curves for different tip radii were calculated using "contact atoms" method (see fig. 9). From this figure one can see that the hardness becomes higher as the tip radius increases. At the maximal depth the hardness values are 20GPa, 27GPa, 29GPa, respectively. Compared with the conic tip, the hardness for blunt tips are higher by $31 \%\left(R=6 a_{0}\right)$ and $40 \%\left(R=12 a_{0}\right)$, respectively. So clearly, the measured hardness will become higher when the tip becomes blunt because of wear.

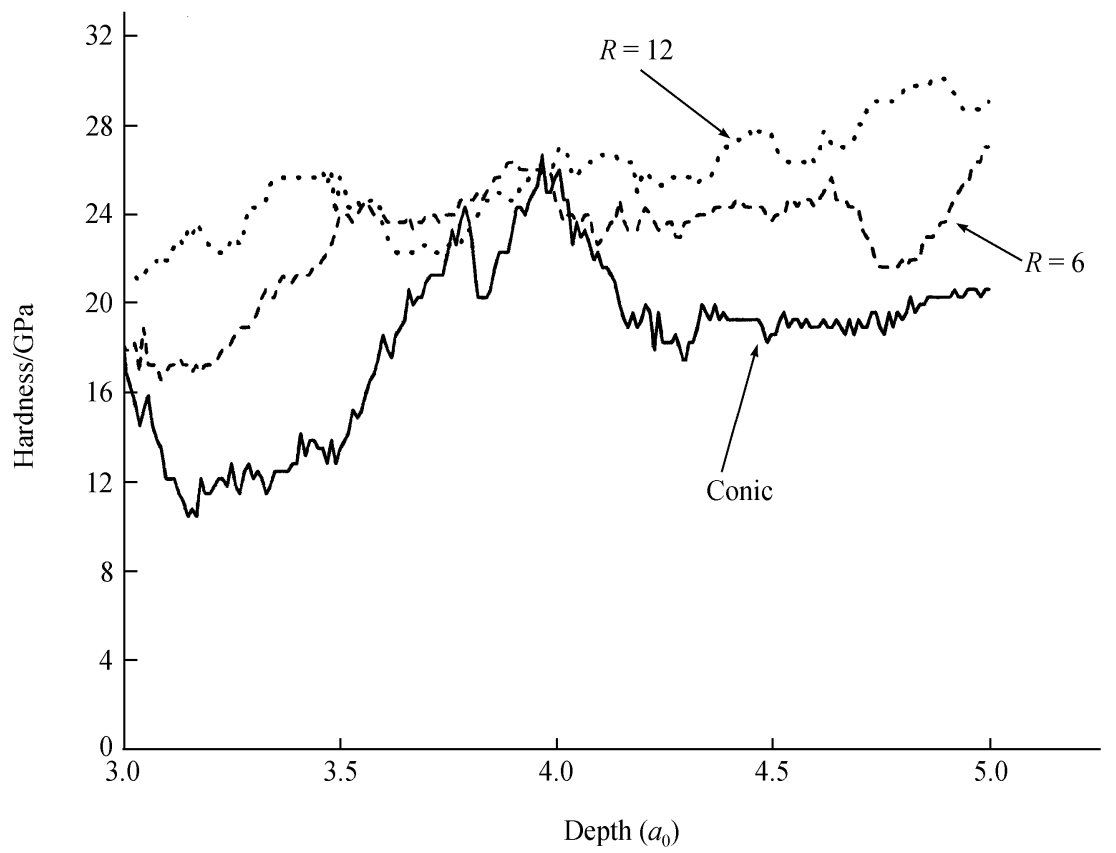

Fig. 9. Hardness versus depth curves calculated by the hybrid representation.

\section{Conclusions and discussion}

MD simulations of shallow nanoindentation in monocrystal copper are performed. In order to obtain the accurate contact area, we proposed a new "contact atoms" method. Specific conclusions of the MD simulation are as follows:

(1) The "contact atoms" method has clear physical implication and can get rid of the approximations made in the calculation of contact area. The method is independent of the penetration depth and the mechanism of the deformation.

(2) Oliver-Pharr method underestimates the contact area of copper nanoindentation, 
and then may overestimate the hardness by as much as $20 \%$.

(3) When the tip becomes blunt because of wear, the measured hardness of materials will become higher.

It can be seen from our simulation that molecular dynamics can clearly clarify the zero point and contact area in nanoindentation and help us understand the mechanical properties at nanoscales better. The molecular dynamics (MD) simulation, which is not limited by sample preparation and testing condition and can be used to simulate various physical and mechanical behaviors in nanoscales, is the most widely used numerical method in nanomechanics. Using molecular dynamics to investigate mechanical problems in nanoscale has a good future.

Acknowledgements This work is supported by the National Natural Science Foundation of China (Grant Nos. 10047006, 10172048, 10232050) and the Special Funds for Major State Basic Research Projects (G2000077305).

\section{References}

1. Lucas, B. N., Oliver, W. C., Swindeman, J. E., The dynamic of frequency-specific, depth-sensing indentation testing, MRS Symposium Proceeding, Vol. 522, San Francisco, California, Pennsylvania: Materials Research Society, 1998, 3-10.

2. Fischer-Cripps, A. C., Nanoindentation, New York: Springer-Verlag, 2002, 61-62.

3. Oliver, W. C., Pharr, G. M., An improved technique for determining hardness and elastic modulus using load and displacement sensing indentation experiments, J. Mater. Res., 1992, 7(6): 1564-1583.

4. Zhang Taihua, Yang Yemin, Developments and applications of nano-hardness techniques, Advances in Mechanics (in Chinese), 2002, 32(3): 349-364.

5. Landman, U., Luedtke, W. D., Burnham, N. A. et al., Atomistic mechanisms and dynamics of adhesion, nanoindentation, and fracture, Science, 1990, 248: 454-461.

6. Leng Yongsheng, Yang Guiping, Hu Yuanzhong et al., Computer experiments on nano-indentation: a molecular dynamics approach to the elasto-plastic contact of metal copper, Journal of Materials Science, 2000, 35: $2061-2067$.

7. Gannepalli, A., Mallapragada, S. K., Molecular dynamics studies of plastic deformation during silicon nanoindentation, Nanotechnology, 2001, 12: 250-257.

8. Cheong, W. C. D., Zhang, L. C., Molecular dynamics simulation of phase transformations in silicon monocrystals due to nano-indentation, Nanotechnology, 2000, 11: 173-180.

9. Christopher, D., Smith, R., Richter, A., Atomistic modeling of nanoindentation in iron and silver, Nnotechnology, 2001, 12: 372-383.

10. Bolshakov, A., Pharr, G. M., Influences of pileup on the measurement of mechanical properties by load and depth sensing indentation techniques, J. Mater. Res., 1998, 13(4): 1049-1058.

11. Li Min, Zhang Tai-hua, Gan Chui-hua et al., Hardness testing on surface layer of material and results analyzing contrastively, Chinese Journal of Aeronautics, 2002, 15(2): 82-89.

12. Daw, M. S., Baskes, M. I., Semiempirical, quantum mechanical calculation of hydrogen embrittlement in metals, Phys. Rev. Lett., 1983, 50(17): 1285-1288.

13. Foiles, S. M.,. Baskes, M. I., Daw, M. S., Embedded-atom-method function for the fcc metals Cu, Ag, Au, Ni, Pd, Pt, and their alloys, Phys. Rev. B, 1986, 33(12): 7983-7991.

14. Ackland, G. J., Tichy, G. I., Vitek V. et al., Simple N-body potentials for the noble metals and nickel, Philosophical Magazine A, 1987, 56(6): 735-756.

15. Zhang Liangchi, Tanaka, H., Towards a deeper understanding of wear and friction on the atomic scale $-\mathrm{a}$ molecular dynamics analysis, Wear, 1997, 211: 44-53.

16. Hockney, R. W., The potential calculation and some applications, Methods in Computational Physics, 1970, 9: $136-211$.

17. Shewchuk, J. R., Triangle: A Two-Dimensional Quality Mesh Generator and Delaunay Triangulator, 1995.

18. Final draft international standard, Metallic materials-Instrumented indentation test for hardness and materials parameters, ISO/FDIS 14577-1:2002(E). 